\title{
A Survey of Energy Efficient Location based Multipath Routing in MANET
}

\author{
Ruchi Gupta \\ M. Tech. Student \\ Department of Computer \\ Engineering, \\ RGPV University, \\ Bhopal, India
}

\author{
Akhilesh A.Waoo \\ Asst. Prof. of M. Tech. \\ Department of Computer \\ Engineering, \\ RGPV University, \\ Bhopal, India
}

\author{
Sanjay Sharma, PhD. \\ Professor \\ Department of Computer \\ Engineering, \\ MANIT, \\ Bhopal, India
}

\begin{abstract}
Mobile Ad hoc network (MANET) is a collection of mobile nodes that communicates with each other in the absence of centralized administration and fixed infrastructure. In MANET each node having a routing capability but their mobility swiftness are different and due to mobile behaviour it is difficult to find out the location of mobile nodes. Due to mobility the routing procedure will affected then the multipath routing protocols are one of best option to reduce the problem of mobility but in multipath routing protocol are phases the problem of flooding. Now Location based routing is one of the ways to find the location of each mobile node through global positioning system and only that location routing procedure will call. But energy is also a one of the main constraint in MANET because if any node having sufficient energy then this one is capable to send, receive and forward the packets in network. This paper grants a qualitative comparison of the approaches in all three areas and examines opportunities for future research.
\end{abstract}

\section{Keywords}

MANET, Mobility, Multipath routing, Location and Energy.

\section{INTRODUCTION}

Mobile ad hoc network are self organised without any administration capability. Majorly Ad hoc networks can be divided into two classes like static and mobile. Static ad hoc networks are the networks where the position of a node may not change once it has become part of the network. Mobile ad hoc networks are the networks in which systems may move arbitrarily. Examples where mobile ad hoc networks may be employed are the establishment of connectivity among vehicles or between handheld devices. Since mobile ad hoc networks change their topology very frequently and without prior notice, routing in such networks is a big task. This research distinguishes two different approaches like topologybased [1] and location or position-based routing. Topologybased routing protocols use the information about the links that exist in the network to perform packet forwarding. They can be further divided into various subclasses proactive, reactive, and hybrid approaches.

Proactive Routing Protocols: Proactive protocols like Optimized Link State Routing (OLSR) [1], Destination Sequenced Distance Vector (DSDV) [2] continuously learn the networks topology by exchanging topological information in between the network nodes. Whenever there is a need for a route to a destination, such route information is immediately provided. If the network topology changes too frequently, the maintenance cost of the network might be very high. The information about actual topology might even not be used, if the network activity is low.

Reactive Routing Protocols: The reactive routing protocols like Dynamic Source Routing protocol (DSR) [3], Ad Hoc on Demand Distance Vector protocol (AODV) [4], Temporally Ordered Routing Protocol (TORA) [5] are based on some sort of query-reply dialog. Reactive protocols proceed for establishing route(s) to the destination only when they require. They do not need periodic transmission of topological information of the network.

Hybrid Routing Protocols: Sometime the reactive or proactive features of a particular routing protocol might not be enough instead a mixture might yield better solution. Hence, in the recent days, several hybrid protocols are proposed like ZRP [6].

Classification of MANET routing protocols could be done on the bases of the methods of delivery of data packets from source to destination as follows:

- $\quad$ Single path Routing Protocols: These are the routing protocols that consider sending information packets to a single destination from a single source. Alternate path is selected in terms of first one break from any reason.

- Multipath Routing Protocols: Multipath routing protocol is a technique that develops the underlying corporal network resources by creating multiple paths from source to destination. If any request fails on single route, the process is again started. But in multipath multiple alternate paths are establish until a new route is established.

Multiple paths also provide route failure protection and load balancing by distributing traffic among a set of disjoint paths if needed.

There are several ways to use and implement the multiple paths. In research [7] and [8], the multiple paths are not used simultaneously. The data packets are transmitted only along one path. Other paths are kept as backup paths in case the used one is broken. When all possible paths are broken, a new multipath detection procedure is initialized again.

AOMDV [9] is one of the multipath routing protocol that offers loop-free extension to AODV. It ensures that alternate paths at every node are disjoint, therefore achieves path disjointness without using source routing. Route tables in AOMDV contain a list of paths for each destination, to support multipath routing. All the paths to a destination have the same destination sequence number. Once a route advertisement with a higher sequence number is received, all routes with the old sequence number are removed. Two additional fields, hop count and last hop, are stored in the 
route table entry to help address respectively the problems of loop freedom and path disjointness. Because the multipath routing protocol implement multipath discovery, the loop freedom guarantee from AODV [4] no longer holds. The hop count field in AOMDV contains the length of the longest path for a particular destination sequence number and is initialized only once, at the time of the first advertisement for that sequence number. Hence, the hop count remains unchanged until a path for a higher destination sequence number is received. It follows that loop freedom is ensured as long as a node never advertises a route shorter than one already advertised, and never accepts a route longer than one already advertised. To ensure that paths in the route table are linkdisjoint, a node discards a path advertisement that has either a common next hop or a common last hop as one already in the route table. This research observed that, as long as each node adheres to this rule, all paths for the same destination sequence number are guaranteed to be link-disjoint. Nodedisjoint paths can be generated with an additional restriction that for a particular destination sequence number, every node always advertises the same designated path to other nodes. Route maintenance in AOMDV is similar. Now this research says that, multipath routing are definitely gives the better performance in the presence of location aware Distance Routing Effect Algorithm for Mobility (DREAM) framework [10], each node maintains a position database that stores position information about each other node that is part of the network. It can therefore be classified as an all-for-all approach. An entry in the position database includes a node identifier, the direction of and distance to the node, as well as a time value that indicates when this information was generated. Of course, the accuracy of such an entry depends on its age. Each node regularly floods packets to update the position information maintained by the other nodes. A node can control the accuracy of its position information available to other nodes by:

- The frequency at which it sends position updates (temporal resolution)

- Indicating how far a position update may travel before it is discarded (spatial resolution)

The temporal resolution of sending updates is coupled with the mobility rate of a node (i.e., the higher the speed, the more frequent the updates). The spatial resolution is used to provide accurate position information in the direct neighbourhood of a node and less accurate information at nodes farther away. The costs associated with accurate position information at very remote nodes can be reduced since; as the authors argue, "the greater the distance separating two nodes, the slower they appear to be moving with respect to each other" (termed the distance effect [11]).

Figure 1 shown below is an example of this "distance effect". Assume that in this example shown below node A is not moving, while nodes $\mathrm{B}$ and $\mathrm{C}$ are moving in the same direction at the same speed. From node A's perspective, the change in direction will be greater for node B than for node C. The distance effect allows low spatial resolution in areas far away from the target node, provided that intermediate hops are able to update the position information contained in the packet.

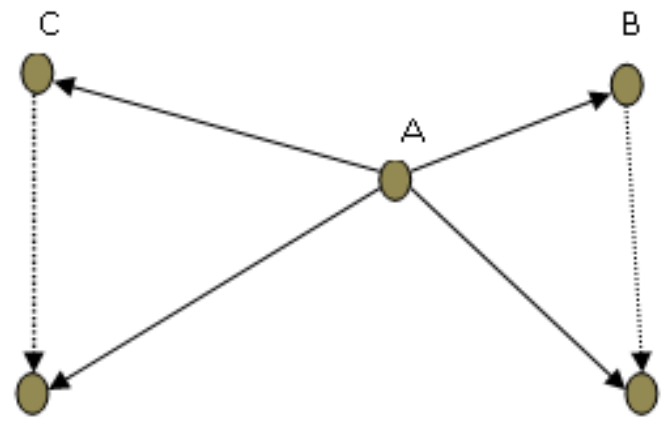

Fig. 1 Distance Effect

In addition of the mentioned routing protocols for MANET, there are some more routing protocols that doesn't rely on any traditional routing mechanisms, instead rely on the location awareness of the participating nodes in the network. Normally, in traditional MANETs, the nodes are addressed only with their IP addresses. On the other end, in case of location-aware routing mechanisms, the nodes are totally aware of their exact physical locations in the threedimensional world. Using Global Positioning System (GPS) this capability might be introduced or with any other geometric methods. On the bases of these concepts, several geocast and location-aware routing protocols have already been proposed. When a node knows about the location of a particular destination, it can direct the packets toward that particular direction from its current position, without using any route discovery mechanism; this is one of the major features of routing protocols. Multipath routing protocols are more battery consuming because always maintained a minimum three alternate paths then in terms of battery constrained single path technique is better but with location based routing definitely multipath protocol consumes less battery power. In MANETs lack of fixed infrastructure and nodes are typically powered by batteries with a limited energy supply wherein each node stops functioning when the battery drains. Energy efficiency is an important consideration in such an environment. Since nodes in MANETs rely on limited battery power for their energy, energy-saving techniques aimed at minimizing the total power consumption of all nodes in the group (minimize the number of nodes used to establish connectivity, minimize the control overhead and so on) and at maximizing the life span should be considered. As a result of the energy constraints placed on the network's nodes, designing energy efficient routing protocols is a crucial concern for MANETs, to maximize the lifetime of its nodes and thus of the network itself [12], [13].

\section{RELATED WORK}

There are several energy-efficient techniques are proposed to reduce energy consumption in MANET. These techniques use energy aware metrics to establish a path in a network. These metrics are transmission power or link distance, residual energy.

LAR (Location Aided routing) uses the location information [14] to reduce routing overhead involved in flooding. LAR uses following schemes for routing:

1. This scheme uses concept of zones that are request zone and expected zone. Before packet transmission it first calculates request zone over which packet is to be transmitted. If the node is in the request zone then it receives and forwards the packet otherwise discard the packet. The same procedure continues 
until expected location is reached where destination node lies.

2. This scheme is based on the estimation of distance from destination. The node having least relative position from destination is chosen to forward packet to its next neighbour until destination is reached.

Brad Karp. H.T. Kung, proposed the theory of GPSR (Greedy perimeter stateless routing) [15] is based on two principles: greedy forwarding and perimeter forwarding. In greedy forwarding it chooses the node that is lying closer to destination and forwards the packet via that node. When packet come to a dead end (means their next hop $=0$ ) then it performs perimeter forwarding in which position using relative neighborhood graph (RNG) is calculated and traversing is done using right hand rule. in this scheme the routing overhead is more.

Gergely et.al has proposed LABAR (Location Area Based Ad hoc Routing) [16] routing protocol is based on virtual backbone formation which is used for representation of position of nodes. G-node (root) initiates virtual backbone formation and tracks the information using GPS which later on helps in directional routing over the network. Source Gnode directs the node of its zone for how to forward packet inside the zone. Each zonal node consults its G-node for best directionality instructions to forward packet to destination node.

Jian Li, Prasant Mohapatra, proposed the concept of LAKER (Location Aided Knowledge Extraction Routing) [17] uses a cached guiding route which helps in assisting the route discovery process. The structure do not change rapidly in this protocol. So it uses guiding locations from source to destination for easy transmission. LAKER request zone helps in reducing routing overhead involved during transmission. Guiding route also helps in finding void areas present over the network.

Geographical protocols uses location information for traversing so these protocol calculate the position of each node using GPS [18,19] (Global positioning system) which requires use of GPS satellite and GPS receiver for communication and the whole process works as follows:

- GPS receiver communicates with GPS satellite for calculating its position.

- $\quad$ Receiver receives the message via satellites.

- Using those signals position is then displayed in the form of latitude and longitude.

Niranjan Kumar Ray and Ashok Kumar Turuk have proposed different energy efficient techniques for wireless ad-hoc network [20]. One of the most commonly used technique is based on reduction of number of route request messages. In second Power control technique, next hop node is chosen depending on the power level of the node. Topology control technique is used to remove the energy-inefficient link from the network by examining the power level of the node. This technique helps network devices to take decision about their transmission range.

Morteza Maleki et.al in [21] have proposed a new poweraware source-initiated (on demand) routing protocol for mobile Ad-hoc networks that increase the network lifetime up to $20 \%$. A greedy policy was applied to fetched paths from the cache to make sure no path would be overused and also make sure that each selected path has minimum battery cost among all possible path between two nodes. Power-aware Source Routing (PSR) has taken care of both the node mobility and the node energy depletion that may cause a path to become invalid.

Energy-based Route Discovery Mechanism in Mobile Ad Hoc Networks [22] selects the route which has lowest energy cost in the network. The cost of energy represents energy consumption of the network in order to prolong all connections between source and destination nodes. The cost of energy is calculated using realistic energy consumption modeling which is used the channel quality to decide whether each packet is successfully received.

Dahai Du et al proposed[23] Location aided Energy-Efficient Routing protocol (LEER) protocol finds out the all possible paths from source to destination and selects minimum energy path to route the packets. The selection of next hop node is based on whether it is situated near to destination than to source as well as transmit power of that node.

An energy aware routing scheme in location based ad-hoc network has proposed by Jangsu Lee et al [24]. This method modifies the LAR protocol in which the virtual grid is applied to ad hoc network region and high energy node is selected as header for each grid which communicates information about nodes in that particular grid. The transmit power of nodes is used to according to the distance between them. The next hop node will be chosen based on transmit power and its distance from the destination.

Nen-Chung Wang et al in [25] have proposed a concept which decides the baseline between the source node and the destination node, for route discovery procedure. The next hop is selected on the bases of baseline by broadcasting the request packets in request zone. The neighboring nodes that are in radio range to the baseline is chosen as the next hop node. This method helps in reduction of control packets overheads by finding a better routing path than LAR scheme. They have proposed a partial reconstruction process for maintaining broken links of routing path.

Arthi Kothandaraman has proposed a protocol which based on transmission power control [26]. It varies the transmission range of a node to exclusively accommodate an independent node's neighbor set. This purely distributed as well as protocol independent scheme and allows low power transmission and preserves connectivity.

ZJ Hass et al in [27] has proposed ZRP (Zonam Routing Protocol) is suitable for wide variety of MANETs, especially for the networks with large span and diverse mobility patterns. In this protocol, each node proactively maintains routes within a local region, which is termed as routing zone. Route creation is done using a query-reply mechanism. For creating different zones in the network, a node first has to know who its neighbours are or the number of nodes that are in the radio range of sender. A neighbour is a node by that sender direct communication can be established connection, and that is, within one hop transmission range of a next node.

Neighbour discovery information is used as a basis for Intrazone Routing Protocol (IARP), which is described in detail in [28] by S.K. Pathan et al. Rather than blind broadcasting, ZRP uses a query control mechanism to reduce route query traffic by directing query messages outward from the query 
source and away from covered routing zones. A covered node is a node which belongs to the routing zone of a node that has received a route query. During the forwarding of the query packet, a node identifies whether it is coming from its neighboring or not. If yes, then it marks all of its known neighbouring nodes in its same zone as covered. The query is thus relayed till it reaches the destination. The destination in turn sends back a reply message via the reverse path and creates the route.

\section{CONCLUSION AND FUTURE WORK}

In order to make possible reliable communication within a MANET, an efficient routing protocol is required to discover routes between mobile nodes. The field of MANETs is rapidly growing due to the many advantages and different application areas. This paper provides the related research information about Multipath routing, Location aware and Energy efficiency factor in MANET. There are many researchers, those are doing a lot of work in these areas which is very useful if the ad hoc network are implemented. Every one idea presents some improvement in field of research with slightly limitations. If due to mobility suddenly node will go out of range then definitely location based multipath routing is better for maintaining the network performance.

For future implementation three modules like location aware, energy and multipath could be combined in MANET. This approach will depends on the results analysis of normal multipath and Location based energy efficient multipath routing protocols. Future work is effective and better than normal multipath routing protocol, it will prove on the base of performance metric. If performance parameter is showing the better result than normal AOMDV then enhancement is better.

\section{REFERENCES}

[1] T. Clausen, et. al, " Optimized Link State Routing Protocol," Internet Draft: draftietf-Manet-olsr- 05.txt, October, 2001.

[2] C. E. Perkins. Ad hoc Networking. Addison-Wesley, Upper Saddle River, NJ, USA, 2001.

[3] D.B. Johnson, D.A.Maltz and Y.Hu, "The Dynamic Source Routing Protocol for Mobile Ad Hoc Networks (DSR)," Internet Draft: draft-ietf-manet-dsr-06.txt, November 2001.

[4] C.E.Perkins, E.M.Royer and S.R. Das, "Ad hoc OnDemand Distance Vector Routing," Internet Draft: draftietf-manet-aodv-09.txt, November 2001.

[5] V.Park and S.Corson, "Temporally-Ordered Routing Algorithm (TORA) Version1 Functional Specification," Internet Draft: draft-ietf-manet-tora-spec-04.txt, July 2001.

[6] Z. Hass, and M. Pearlman, "The performance of query control scheme for the zone routing protocol", in Proc. ACM SIGCOMM, Aug. 1998.

[7] J. Broch, D. Johnson, and D. Maltz, "The Dynamic Source Routing Protocol for Mobile Ad hoc Networks," http://www.ietf.org/internet-drafts/draft-ietfmanet- dsr01.txt, IETF Internet Draft Dec 1998.

[8] Nasipuri and S. R. Das, "On-Demand Multipath Routing for Mobile Ad Hoc Networks," Proceedings of the 8th Int. Conf. On Computer Communications and Networks (IC3N), Boston, October 1999.
[9] M. K. Marina and S. R. Das, "Ad hoc On-demand Multipath Distance Vector Routing," Computer Science Department, Stony Brook University, 2003.

[10] S. Basagni et al., "A Distance Routing Effect Algorithm for Mobility (Dream)," Proc. 4th Annual ACM/IEEE Int. Conf. Mobile Computing and Networking, MOBICOM '98, pp. 76-84,Dallas, TX, USA, 1998.

[11] S. Basagni, I. Chlamtac, and V. Syrotiuk, "Geographic Messaging in Wireless Ad Hoc Networks," Proc. 49th IEEE Int'l. Vehic. Tec. Conf., Houston, TX, USA, vol. 3, pp. 1957-61, 1999.

[12] G. Girling, J. Li Kam Wa, P. Osborn, R. Stefanova, “The Design and Implementation of a Low Power Ad Hoc Protocol Stack”, IEEE Personal Communications, 2000.

[13] Y. Hu, D. Johnson, A. Perrig, "SEAD: Secure Efficient Distance Vector Routing for Mobile Wireless Ad hoc Networks", vol. 1, no. 1, pp. 175-192, July 2003.

[14] Young B. Ko, Nitin H. Vaidya, "Location-aided Routing (LAR) in mobile ad hoc networks", ACM/Baltzer Wireless networks (WINET), Vol. 6-4 pp 307-321 2000.

[15] Brad Karp. H.T. Kung, "GPSR: Greedy Perimeter Stateless Routing for Wireless networks", Proc. ACM MobiCom 2000.

[16] Gergely et.al, "LABAR: Location Area Based Ad Hoc Routing for GPS-Scarce Wide-Area Ad Hoc Networks", IEEE Computer Society, 2003.

[17] Jian Li, Prasant Mohapatra, "LAKER: Location Aided Knowledge Extraction Routing for Mobile Ad Hoc Networks", Proc. IEEE Wireless Comm. and Networking Conf. (WCNC '03), 2003.

[18] NAVSTAR GPS operations, available via WWW at URL: http://tycho.usno.navy.mil/gpsinfo.html.

[19] B.W. Parkinson and S.W. Gilbert, NAVSTAR: global positioning system - ten years later, Proceedings of the IEEE 71(10) (1983).

[20] Niranjan Kumar Ray \& Ashok Kumar Turuk, "Energy Efficient Techniques for Wireless Ad Hoc Network", International Joint Conference on Information and Communication Technology, pp105-111, 2010.

[21] Morteza Maleki, Karthik Dantu \& Massoud Pedram, "Power-aware Source Routing Protocol for Mobile Ad Hoc Networks", Iternational Symposium on Low Power Electronics and Design (ISLPED), pp72-75, 2002

[22] J. Kanjanarot, K. Sitthi, and C \& Saivichit,"Energybased Route Discovery Mechanism in Mobile Ad Hoc Networks", ICA0T2006, pp1967-1972, 2006

[23] Dahai Du \& Huagang Xiong, "A Location aided EnergyEfficient Routing Protocol for Ad hoc Networks", 19th Annual Wireless and Optical Communications Conference (WOCC), pp1-5, 2010.

[24] Jangsu Lee, Seunghwan Yoo \& Sungchun Kim, "Energy aware Routing in Location based Ad-hoc Networks", Proceedings of the 4th International Symposium on Communications, Control and Signal Processing (ISCCSP), pp3-5, 2010.

[25] Nen-Chung Wang \& Si-Ming Wang, “An Efficient Location-Aided Routing Protocol for Mobile Ad Hoc Networks", 11th International Conference on Parallel 
and Distributed Systems (ICPADS'05), Vol. 1, pp335341,2010 .

[26] Arthi Kothandaraman, “An Energy-Efficient Transmission Power Control Protocol for Cooperative Robotics", Master Thesis, Auburn University, Alabama, pp1-63, 2010.

[27] Haas ZJ, Pearlman MR, Samar P "The Zone Routing Protocol (ZRP) for Ad Hoc Networks" IETF draft, July
2002, available at http://tools.ietf.org/id/draft-ietfmanetzone-zrp-04.txt.

[28] A.-S.K. Pathan and C.S. Hong Haas ZJ, Pearlman MR, Samar P "Intrazone Routing Protocol (IARP)" IETF Internet Draft, July 2002, available at http://tools.ietf.org/wg/manet/draft-ietf-manetzoneierp/draft-ietf-manetzone-rp-02-from-01.diff.txt. 